\title{
Probing the intravascular and interstitial compartments of remodeled myocardium in heart failure patients with preserved and reduced ejection fraction: a CMR study
}

\author{
Pier Giorgio Masci ${ }^{1 *}$ (D, Anna Giulia Pavon ${ }^{1,2}$, Gregoire Berchier $^{3}$ and Juerg Schwitter ${ }^{1}$
}

\begin{abstract}
Background: Recent autopsy studies found microvascular rarefaction in remodeled myocardium of patients who died of heart failure with preserved ejection-fraction (HFpEF). This condition has not been investigated so far by non-invasive methods in patients with HFpEF. The aim was to quantify the intravascular volume (IV) compartment by CMR in HFpEF patients.

Methods: In two separate CMR examinations, HFpEF patients ( $n=6 ; 12$ examinations) and post-myocardial infarction patients (post-Ml; $n=6 ; 12$ examinations) were studied with $\mathrm{T}_{1}$-mapping (MOLLI-sequence) before and after IV bolus of $0.03 \mathrm{mmol} / \mathrm{Kg}$ of the intravascular contrast-medium (CM) Gadofosveset and $0.2 \mathrm{mmol} / \mathrm{Kg}$ of the extravascular CM Gadobutrol yielding IW and extracellular volume (ECV), respectively. Healthy controls ( $n=10$ with Gadofosveset only, $n$ $=10$ with Gadobutrol only) were also studied with the same protocol. IW and ECV were measured in the basal septum (without ischemic scar in post-Ml patients). In post-MI patients, ECV and IW were also measured in the ischemic scar. Left ventricular (LV) volumes, mass, and ejection-fraction were measured by standard protocol. LV global longitudinal strain (GLS) was calculated by feature tracking on long-axis cine acquisitions.
\end{abstract}

Results: LV mass to end-diastolic volume ratio and GLS in HFpEF were higher and lower, respectively, than in healthy controls and post-MI patients, whereas the post-Ml patients showed lower LV ejection-fraction. Compared to healthy myocardium of controls, IW in scar was reduced $(0.135 \pm 0.018$ vs $0.109 \pm 0.008$, respectively, $p=0.005)$, while ECV was increased $(0.244 \pm 0.037$ vs $0.698 \pm 0.106$, respectively, $p<0.001)$. However, IW did not differ among HFpEF, post-MI, and healthy controls $(0.155 \pm 0.033,0.146 \pm 0.038$, and $0.135 \pm 0.018$, respectively, $p=0.413)$, whereas ECV was higher in $\mathrm{HFpEF}$ than in post-Ml and healthy controls $(0.304 \pm 0.159,0.270 \pm 0.017$, and $0.244 \pm 0.037$, respectively, $p=0.003)$.

Conclusions: The $\mathrm{T}_{1}$-mapping technique combined with an intravascular $\mathrm{CM}$ shows potential to measure IW. In infarct scar with substantially increased ECV, IW was significantly reduced. Unlike in infarct scar, in remodeled myocardium of HFpEF patients, increased ECV was not accompanied by a reduction of IW.

\section{Introduction}

Heart failure with preserved ejection fraction (HFpEF) is a common condition with increasing prevalence and high morbidity and mortality $[1,2]$ HFpEF occurs in association with advanced age, cardiovascular and metabolic comorbidities as well as with a pro-inflammatory state [2]. It is characterized by impaired left ventricular (LV)

\footnotetext{
*Correspondence: pgmasci@gmail.com; pier-giorgio.masci@chuv.ch ${ }^{1}$ Lausanne University Hospital, Cardiovascular Department, and University of Lausanne, Lausanne, Switzerland

Full list of author information is available at the end of the article
}

relaxation and increased diastolic stiffness [2, 3]. Despite intensive research, the myocardial abnormalities subtending HFpEF are incompletely understood. Studies of endomyocardial biopsy or of autopsy of selected HFpEF patients reported myocyte hypertrophy, diffuse interstitial fibrosis and signs of systemic and myocardial inflammation $[4,5]$. In this setting microvasculature rarefaction was also observed, and it was inversely correlated to myocardial fibrosis [6, 7]. Accordingly, the detection and quantification of microvascular rarefaction might be of great value for assessing the pathophysiological abnormalities

(c) The Author(s). 2019 Open Access This article is distributed under the terms of the Creative Commons Attribution 4.0 International License (http://creativecommons.org/licenses/by/4.0/), which permits unrestricted use, distribution, and reproduction in any medium, provided you give appropriate credit to the original author(s) and the source, provide a link to the Creative Commons license, and indicate if changes were made. The Creative Commons Public Domain Dedication waiver (http://creativecommons.org/publicdomain/zero/1.0/) applies to the data made available in this article, unless otherwise stated. 
occurring in patients with $\mathrm{HFpEF}$ and for establishing its diagnosis. However, the non-invasive measurement of myocardial intravascular compartment in humans is challenging.

Based on these premises, we developed a cardiac magnetic resonance (CMR)-based approach for the quantification of the myocardial intravascular volume (IVV) consisting in the use of an intravascular gadolinium-based contrast medium (CM) combined with the quantitative measurement of myocardial $\mathrm{T}_{1}$ by currently available mapping technique $[8,9]$. To this end, we administered an intravascular and extravascular CM 1). in healthy controls, 2). in HFpEF patients with LV hypertrophy (LVH) and/or proven interstitial myocardial fibrosis, and 3). in patients with chronic myocardial infarctions (MI). Post-MI patients were included to study the remote non-infarcted myocardium, which typically undergoes remodelling processes with eccentric hypertrophy [10]. In addition, in post-MI patients, we tested the hypothesis that the intravascular CM allows to quantify the reduced IVV, which is a typical feature of post-ischemic infarct scar [11].

\section{Methods}

\section{Study population}

Twenty healthy controls without cardiovascular risk factors, $6 \mathrm{HFpEF}$ patients and 6 patients with previous MI were enrolled into the study. The healthy controls were recruited through local advertising and had no history or symptoms of cardiovascular disease and no cardiovascular risk factors. Patients were recruited from the clinical database of our CMR center after having performed a clinically-indicated CMR examination using an extravascular CM (Gadobutrol, Gadovist ${ }^{\circ}$, Bayer Healthcare, Germany). HFpEF patients were selected according to the following criteria: 1) LV-EF $>40 \%$ and presence of left ventricular hypertrophy ( $\mathrm{LVH})$ and/or $\mathrm{T}_{1}$-mapping based extracellular volume fraction $(\mathrm{ECV})>28 \%$, [8]; 2) dyspnea (unless physical activity was compromised by peripheral arterial disease); 3) history of arterial hypertension and $\geq 1$ additional cardiovascular risk factor. Exclusion criteria were myocardial ischemia and/or previous MI (by clinical history and on CMR) or known significant valvular heart disease. Post-MI patients were selected on the basis of previous MI (>12 months) and chronic fibrotic infarct scar on post-contrast CMR. In this subgroup, the scar did not involve the basal interventricular septum. Extensive validation of the $T_{1}$-mapping technique has been performed with regard to standardization of the method [12-14] and in comparison to histopathology when combined with an extracellular Gd-based CM to measure ECV [15]. In this study, we aimed to expand the $T_{1}$ mapping technique to measure the distribution volume of an intravascular $\mathrm{CM}$ in order to obtain IVV non-invasively. Accordingly, ECV and IVV were measured by the administration of the extravascular CM gadovist and the intravascular CM gadofosveset (Ablavar', Lantheus Medical Imaging, US), respectively. In the controls, the two CMs were administered in two separate groups of 10 controls each (to avoid $2 \mathrm{CM}$ administrations in the same subject according to the Local Ethic Committee recommendation). Post-MI and HFpEF patients were studied in a second research-dedicated CMR examination with the administration of the intravascular CM.

The study complied with the Declaration of Helsinki and all examinations were conducted at the Lausanne University Hospital after obtaining the approval from local ethics committee. All study participants provided written informed consent.

\section{Cardiac magnetic resonance protocol}

In the extravascular CMR protocol, i.e. in the clinical CMR examination, cine SSFP images were acquired for functional LV evaluation, followed by pre-contrast $\mathrm{T}_{2}$-map and $\mathrm{T}_{1}$-map acquisitions at the basal short-axis position. At $\geq 15$-min after the i.v. bolus administration of $0.20 \mathrm{mmol} / \mathrm{kg}$ of the extracellular $\mathrm{CM}$ gadobutrol, the $\mathrm{T}_{1}$-map was re-acquired at the same short-axis basal position. Then, late gadolinium enhancement (LGE) imaging was performed on short axis covering the entire LV.

In the intravascular CMR protocol, cine SSFP and $\mathrm{T}_{2}$-map and native $\mathrm{T}_{1}$-maps were acquired as in the extracellular CMR protocol. Then, at $3 \mathrm{~min}$ after the i.v. bolus administration of $0.03 \mathrm{mmol} / \mathrm{kg}$ of the intravascular $\mathrm{CM}$ gadofosveset, the $\mathrm{T}_{1}$-map was re-acquired at the same basal short-axis slice position. The 3-min interval was chosen to allow for a homogeneous distribution of the intravascular CM in the subjects' blood pool, i.e. in the intravascular compartment. This protocol was performed in all patients ( $6 \mathrm{HFpEF}$ and 6 post-MI patients) and in 10 healthy controls (as described above). In the extravascular and intravascular CMR protocol, hematocrit was measured immediately before CMR.

All studies were performed on a 1.5 Tesla system (Siemens Healthcare, Aera Magnetom, Erlangen, Germany) using dedicated cardiac software, a 32-channel phased array receiver coil, and ECG triggering. The pulse sequence parameters for functional cine SSFP, $\mathrm{T}_{1}$ - and $\mathrm{T}_{2}$-mapping as well as for the LGE imaging are given in Table 1.

\section{Image analysis}

The formula- 1 below was applied to calculate the CM distribution volume after administration of the extra-cellular and intravascular CM yielding extracellular volume (ECV) and intravascular volume (IVV), respectively [8]. 
Table 1 CMR pulse sequence parameters

\begin{tabular}{|c|c|c|c|}
\hline Tecnique & Sequence & Parameters & Notes on Image Generation \\
\hline Cine imaging & $\begin{array}{l}\text { Breath-hold segmented steady } \\
\text { state free precession }\end{array}$ & $\begin{array}{l}\text { flip angle:56 } 6^{\circ} \text { voxel size: } 1,4 \times 1,4 \times 6,0 \\
\text { TR/TE:37,1/1,19 ms, matrix: } 256 \times 208 \text {; field-of- } \\
\text { view: } 300 \times 240 \mathrm{~mm}^{2} \text {; parlallel imaging acquisi- } \\
\text { tion technique: } 2 \text {; cardiac phases: } 25\end{array}$ & \\
\hline T1-mapping & $\begin{array}{l}\text { 2D Breath-hold MOdified Look- } \\
\text { Locker Inversion-Recovery (MOLLI) }\end{array}$ & $\begin{array}{l}\text { Acquisition Sampling Scheme: } \\
\text { a) pre-contrast (native): } 5(3) 3 \\
\text { b) Post-contrast: } 4(1) 3(1) 2 \\
\text { Read-out: SSFP single-shot, trigger delay in } \\
\text { mid-diastole, flip angle: } 35^{\circ} \text {, voxel size: } 1.5 \times \\
1.5 \times 8 \mathrm{~mm}^{3} ; \text { parallel acquisition factor: } 2 \text {, number } \\
\text { of inversion pulse } 2 \text { and } 3 \text { for native and post- } \\
\text { contrast }\end{array}$ & $\begin{array}{l}\text { Generation of inline motion corrected pixel- } \\
\text { based T1-maps by acquiring a series of images } \\
\text { over several heart-beats with shifted inversion } \\
\text { delays corrected by RR duration }\end{array}$ \\
\hline T2-mapping & $\begin{array}{l}\text { 2D Breath-hold T2-prepared SSFP } \\
\text { sequence }\end{array}$ & $\begin{array}{l}\text { Read-out: single-shot SSFP, trigger delay in } \\
\text { mid-diastole, flip angle: } 70^{\circ} \text {, voxel size: } 1.9 \times \\
1.9 \times 8 \mathrm{~mm}^{3} \text {, matrix size: } 192 \times 154 \text {; FOV: } \\
360 \times 288 \mathrm{~mm}^{2} \text { parlallel imaging acquisition } \\
\text { technique: } 2\end{array}$ & $\begin{array}{l}\text { Generation of inline motion corrected pixel- } \\
\text { based T2-maps by acquiring } 3 \text { images with dif- } \\
\text { ferent T2 preparation time }(0,25,55 \text { ms) with a } \\
\text { gap of } 2 \text { RR interval within } 1 \text { breath-hold }\end{array}$ \\
\hline $\begin{array}{l}\text { Late } \\
\text { gadolinium } \\
\text { enhancement }\end{array}$ & $\begin{array}{l}\text { 2D breath-hold segmented } \mathrm{T1-} \\
\text { weighted gradient echo inversion } \\
\text { recover with phase sensative } \\
\text { reconstraction }\end{array}$ & $\begin{array}{l}\text { Flip angle: } 35^{\circ} \text {; voxel size: } 1,3 \times 1,3 \times 8 \mathrm{~mm}^{3} \text {; } \\
\text { matrix size; } 256 \times 208 \text {; TR.TE:848/3,8 ms; FOV: } \\
340 \times 275 \mathrm{~mm}^{2} \text {; parallel acquisition factor } 2 \text {, } \\
\text { number of segment: } 20 \text { to } 30\end{array}$ & \\
\hline
\end{tabular}

\section{Distribution volume $=(1-$ hematocrit $)$}

$$
\times \frac{(1 / \text { T1myo-post })-(1 / \text { T1myo-pre })}{(1 / \text { T1blood-post })-(1 / \text { T1blood-pre })}
$$

$\mathrm{T}_{1 \text {-myo-pre }}$ and $\mathrm{T}_{1 \text {-blood-pre }}$ indicate myocardial and blood native $T_{1}$ values, respectively, and $T_{1-\text { myo-post }}$ and $\mathrm{T}_{1 \text {-blood-post }}$ indicate the $\mathrm{T}_{1}$ values of myocardium and blood pool after CM administration, respectively.

ECV and IVV were measured 1). in normal myocardium of controls, i.e. in the basal septum; 2). in the basal septum of HFpEF patients, and 3). in the basal septum (=non-infarcted myocardium) and in the infarcted (=LGE positive) myocardium of the post-MI patients (Fig. 1). The interstitial space was calculated as the difference between ECV and IVV.

All studies were saved in DICOM format and analyzed by 1 operator with $>10$ years experience in CMR using GTVolume software (GyroTools ${ }^{\mathrm{Tm}}$, Version 2.2.1, Zurich, Switzerland). For all maps acquired in the basal short-axis, a region of interest (ROI) was manually drawn in the mid-layer of the interventricular septum (segment 2 and 3 according to AHA segmentation). The ROI was drawn by leaving $2 \mathrm{~mm}$ from each endocardial side to avoid partial volume contamination with blood pool signal. On $\mathrm{T}_{1}$-maps, a second ROI was manually traced in the LV cavity avoiding papillary muscles and trabeculas. These myocardial and blood pool pre-contrast ROIs were then copied to the post-contrast $\mathrm{T}_{1}$-maps. Additionally, for post-MI patients $(n=6)$, another ROI was drawn in the infarcted region in the native and corresponding post-contrast $\mathrm{T}_{1}$-maps. For a correct identification of the fibrotic scar on the $\mathrm{T}_{1}$-maps, a careful side-by-side comparison between $\mathrm{T}_{1}$-maps and LGE images was performed. In LGE positive patients the scar mass was quantified as myocardium with a signal intensity exceeding the mean signal intensity of remote myocardium by $>6 \mathrm{SD}$ using a semi-automatic algorithm [16] and expressed as mass (g) or as percentage of LV mass (\%).

\section{Feature tracking analysis}

Two-dimensional Cardiac Performance Analysis Software (TomTec, Munich, Germany) was used to obtain global LV longitudinal strain derived as the mean between longitudinal strain obtained in 2-chamber, 3-chamber, and 4-chamber cine data sets. The mathematical assumptions used and the clinical validation of the feature tracking technology have been previously described [17]. After the upload of the CMR images on a dedicated workstation, the endocardial border of the LV was manually traced in the end-diastolic frame and the software automatically propagated the contour and followed its features throughout the remainder of the cardiac cycle.

\section{Statistical analysis}

Continuous variables are expressed as mean value \pm SD and categorical variables are expressed as frequency and (\%). Continuous variable differences across healthy controls, HFpEF, and post-MI patients were analyzed by ANOVA, and followed by Bonferroni's post hoc analyses when required. Categorical variable differences across the groups were assessed by Chi-squared, or by Fisher's exact test if the expected cell count was $<5$. Differences in $p$ value $<0.05$ were considered statistically significant. All analyses were performed using SSPS version 21 (SPSS, Chicago, Ill-US). 

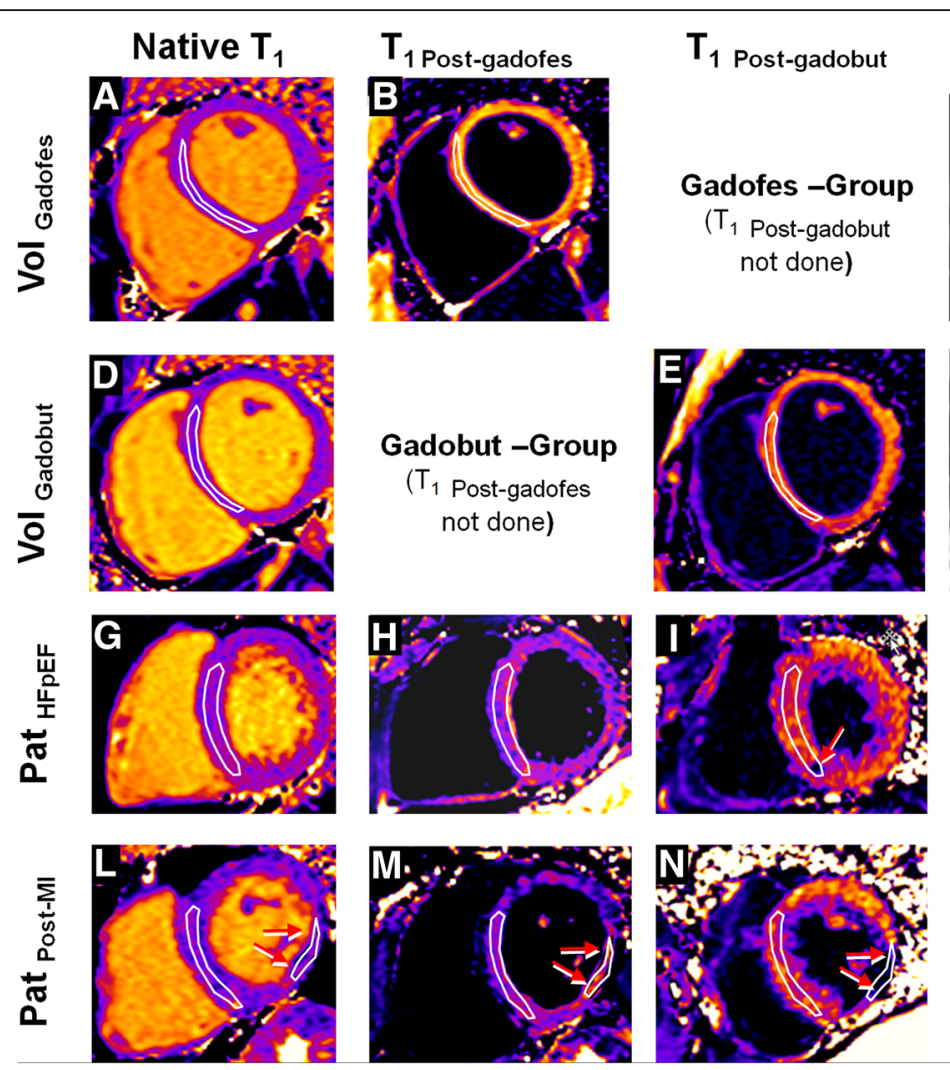

LGE
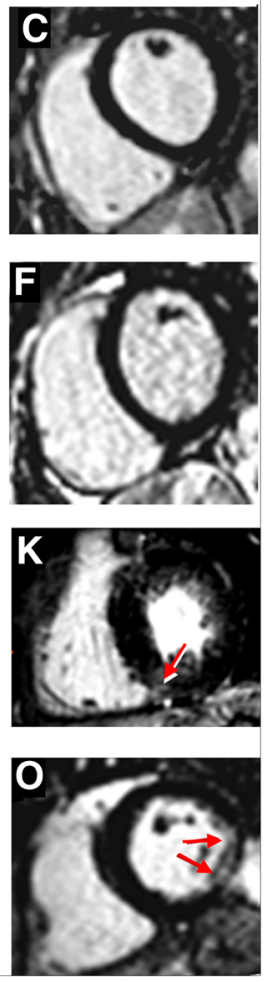

Fig. 1 Native and post-contrast $T_{1}$-maps in healthy volunteers, HFpEF, and post-Ml patients. First and second rows, basal short-axis native (a, d), post-gadofosveset (b), post-gadobutrol (e) $T_{1}$-maps and LGE image $(\mathbf{c}, \mathbf{f})$ in two healthy volunteers. The region-of-interest (ROI) was traced within the basal interventricular septum in all volunteers and patients. Third and fourth rows, in HFpEF and post-MI patients, respectively, show basal short-axis native $(\mathbf{g}, \mathbf{I})$, post-gadofosveset $(\mathbf{h}, \mathbf{m})$, post-gadobutrol $(\mathbf{I}, \mathbf{n}) \mathrm{T}_{1}$-maps, and LGE images $(\mathbf{k}, \mathbf{o})$. In the HFpEF patient (third row), nonischemic scars (red arrow in K) was included in the septal ROI for the calculation of IW and ECV. In post-Ml patients (bottom row), a second ROI was traced within the ischemic scar taking as reference the LGE image (o, with red arrows indicating the scar)

\section{Results}

\section{Characteristics of healthy controls and patients}

The baseline characteristics of the healthy controls and patients are summarized in Table 2. Healthy controls were younger and did not have risk factors as compared to patients. For CMR characteristics, LV volumes did not differ significantly across the groups, whereas LV-EF was consistently lower in patients with previous MI than in healthy controls. In the HFpEF group, LV-EF was preserved with $58 \pm 10 \%$ [18] with only 1 patient with a LV-EF $<50 \%$, i.e. $48 \%$ ). LV mass and the ratio between LV mass and LV end-diastolic volume were greater in the HFpEF group than in controls and post-MI patients.

\section{Distribution volume of intravascular CM in normal myocardium and post-MI scar tissue}

In healthy controls, by applying formula-1, a low distribution volume in the myocardium of $0.135 \pm 0.018$ was obtained after intravascular $\mathrm{CM}$ administration, reflecting the IVV of normal myocardium. In the scar tissue of post-MI patients, IVV was reduced as compared to healthy controls $(0.109 \pm 0.008$ vs $0.135 \pm 0.018, p=0.005$; (Fig. 2a) likely representing the well-known microvascular rarefication in scar tissue, a hallmark of dense fibrotic tissue [11]. The opposite was found for ECV, which was massively elevated in the scar tissue in comparison to normal healthy myocar$\operatorname{dium}(0.698 \pm 0.106$ vs $0.244 \pm 0.037, p<0.001$; Fig. $2 b)$.

\section{Distribution volume of intra- and extravascular CM in non-infarcted myocardium of healthy controls and patients}

Native $T_{1}$ value of the myocardium was slightly lower in controls than in HFpEF or post-MI patients, whereas native $T_{1}$ of blood and hematocrit were comparable among groups (Table 2). The IVV of the basal septum did not differ between healthy controls, post-MI and HFpEF patients (Table 2, Fig. 2c. By pooling the IVV data of the basal septum of post-MI and HFpEF patients $(n=12)$, IVV of the basal septum tended to be higher in patients than in healthy controls $(0.151 \pm 0.034$ vs $0.135 \pm 0.018, p=0.216)$. On the other hand, ECV of the basal septum was significantly increased in HFpEF and post-MI patients as compared to the controls $(0.301 \pm 0.052$ vs $0.276 \pm 0.023$ vs $0.231 \pm 0.029$, respectively, over-all $p<0.001$, Fig. $2 \mathrm{~d}$ ). 
Table 2 Baseline characteristics and CMR results

\begin{tabular}{|c|c|c|c|c|c|}
\hline Variable & $\begin{array}{l}\text { Volunteer Gadofesveset } \\
(n=10)\end{array}$ & $\begin{array}{l}\text { Volunteer Gadobutrol } \\
(n=10)\end{array}$ & $\begin{array}{l}\text { HFpEF } \\
\text { Patients } \\
(n=6)\end{array}$ & $\begin{array}{l}\text { Post-Ml } \\
\text { Patients } \\
(n=6)\end{array}$ & $\begin{array}{l}\text { Overall } \\
p \text {-value }\end{array}$ \\
\hline $\mathrm{Age}^{\mathrm{a}}$ & $35 \pm 15$ & $36 \pm 11$ & $69 \pm 10$ & $58 \pm 13$ & $<0.001$ \\
\hline BMl & $23 \pm 1$ & $24 \pm 3$ & $26 \pm 3$ & $27 \pm 3$ & 0.099 \\
\hline Gender (male) n, \% & $7(70)$ & $7(70)$ & $5(83)$ & $4(67)$ & 0.771 \\
\hline Dyspnea $(\%)^{\mathrm{e}}$ & n.a & n.a & $4(67 \%)$ & $0(0)$ & 0.014 \\
\hline Symptomtic PAD & n.a & n.a & $2(33 \%)$ & $0(0)$ & \\
\hline Hypertension n, (\%) & n.a & n.a & $6(100)$ & $5(83)$ & 0.296 \\
\hline Hypercholesterolemia n, (\%) & n.a & n.a & $4(67)$ & $3(50)$ & 0.558 \\
\hline Active Smoking n, (\%) & n.a & n.a & $4(67)$ & $3(50)$ & 0.557 \\
\hline Family for CAD n, (\%) & n.a & n.a & $1(17)$ & $1(17)$ & NA \\
\hline Diabetes n (\%) & n.a & n.a & $2(33)$ & $0(0)$ & 0.455 \\
\hline LVEDVI (ml/m2) & $87 \pm 14$ & $84 \pm 10$ & $79 \pm 16$ & $95 \pm 16$ & 0.247 \\
\hline LVESVI (ml/m2) & $33 \pm 7$ & $33 \pm 5$ & $44 \pm 26$ & $52 \pm 17$ & 0.064 \\
\hline LVEF $(\%)^{d}$ & $63 \pm 6$ & $61 \pm 12$ & $58 \pm 10$ & $46 \pm 11$ & 0.002 \\
\hline LVMI $\left(g / \mathrm{m}^{2}\right)^{\mathrm{b}}$ & $60 \pm 11$ & $61 \pm 17$ & $85 \pm 11$ & $73 \pm 16$ & 0.009 \\
\hline LVM/LVEDV c, b & $0.70 \pm 11$ & $0.72 \pm 0,17$ & $1.10 \pm 0.15$ & $0.77 \pm 0.14$ & $<0.001$ \\
\hline Global Longitudinal Strain (\%) ${ }^{\mathrm{b}}$ & -22.78 & -21.52 & -16.43 & -17.07 & 0.015 \\
\hline Ischemic Scar n, (\%) ${ }^{f}$ & n.a & n.a & n.a & $6(100)$ & n.a \\
\hline Ischemic Scar Extent (\% LV mass) ${ }^{f}$ & n.a & n.a & n.a & $8.4 \pm 2.9$ & n.a. \\
\hline Native $T_{1}$ septum (ms) ${ }^{a}$ & $1000 \pm 19$ & $989 \pm 27$ & $1028 \pm 31$ & $1029 \pm 44$ & 0.029 \\
\hline Native $T_{1}$ blood (ms) & $1568 \pm 85$ & $1601 \pm 110$ & $1607 \pm 101$ & $1474 \pm 257$ & 0.317 \\
\hline $\mathrm{T}_{2}$ septum (ms) & $43.8 \pm 1.4$ & $45.3 \pm 1.8$ & $46.2 \pm 1.0$ & $44.7 \pm 3.0$ & 0.099 \\
\hline Hct & $0.43 \pm 0.04$ & $0.043 \pm 0.06$ & $0.40 \pm 0.06$ & $0.43 \pm 0.04$ & 0.726 \\
\hline ECV septumb & n.a. & $0.244 \pm 0.037$ & $0.304 \pm 0.0159$ & $0.270 \pm 0.017$ & 0.003 \\
\hline IW septum & $0.135 \pm 0.018$ & n.a. & $0.155 \pm 0.033$ & $0.146 \pm 0.038$ & 0.413 \\
\hline Interstitial Space & n.a & n.a & $0.149 \pm 0.033$ & $0.124 \pm 0.044$ & 0.281 \\
\hline
\end{tabular}

${ }^{\mathrm{a}}$ Bonferroni post-hoc analysis with $p<0.05$ between post-MI and HFpEF versus the controls; ${ }^{\mathrm{b}}$ Bonferroni post-hoc analysis with $p<0.05$ between $\mathrm{HFpEF}$ versus the controls; ' ${ }^{\mathrm{C}}$ Bonferroni post-hoc analysis with $p<0.05$ between HFpEF versus post-MI patients; ${ }^{\mathrm{d} B o n f e r r o n i}$ post-hoc analysis with $p<0.05$ between post-MI patients versus controls; 1 patient in the HFpEF group with $\mathrm{EF}<50 \%(=48 \%)$. ${ }^{\mathrm{e}}$ patients were limited by peripheral arterial disease; ${ }^{\mathrm{f}} \mathrm{Scar}$ on late gadolinium enhancement. Hct: hematocrit; PAD: peripheral arterial disease

The $\mathrm{T}_{2}$ values of the septum tended to be higher in HFpEF than post-MI patients or controls (Table 2), but were within the upper normal range $(48.0 \mathrm{~ms})$ defined as mean plus 2 standard deviations in the pooled data of the healthy controls $\left(n=20, T_{2}=44.6\right.$ $\pm 1.7 \mathrm{~ms}$ ).

\section{Discussion}

The main findings of the current study can be summarized as follows. First, in patients with HFpEF, there was no reduction of the myocardial intravascular compartment as derived by $\mathrm{T}_{1}$-mapping after gadofosveset administration, while the extracellular compartment was expanded as compared to controls. Second, in the scar tissue of post-MI patients the intravascular compartment was significantly reduced, while the extracellular compartment was massively increased. Third, myocardial edema was not detected in the remodeled myocardium of HFpEF patients as evidenced by the normal $\mathrm{T}_{2}$ values.

By assuming that the vascular endothelium is not permeable to albumin under normal conditions, gadofosveset, which binds reversibly to albumin, behaves preferentially as an intravascular CM after intravenous bolus injection. Using $\mathrm{T}_{1}$-mapping, we calculated the relaxivity of myocardium and blood pool immediately, i.e. at $3 \mathrm{~min}$, after gadofosveset bolus injection, and these measurements, in conjunction with the hematocrit, were used to derive the myocardial IVV by adopting the previously published formula $[9,11]$. The IVV was significantly decreased in the myocardial scar of post-MI patients as compared to normal myocardium of healthy controls, indicating that this approach is able to detect a reduced intravascular space, which is a key feature of dense fibrotic scar [11]. When estimating IVV by the 

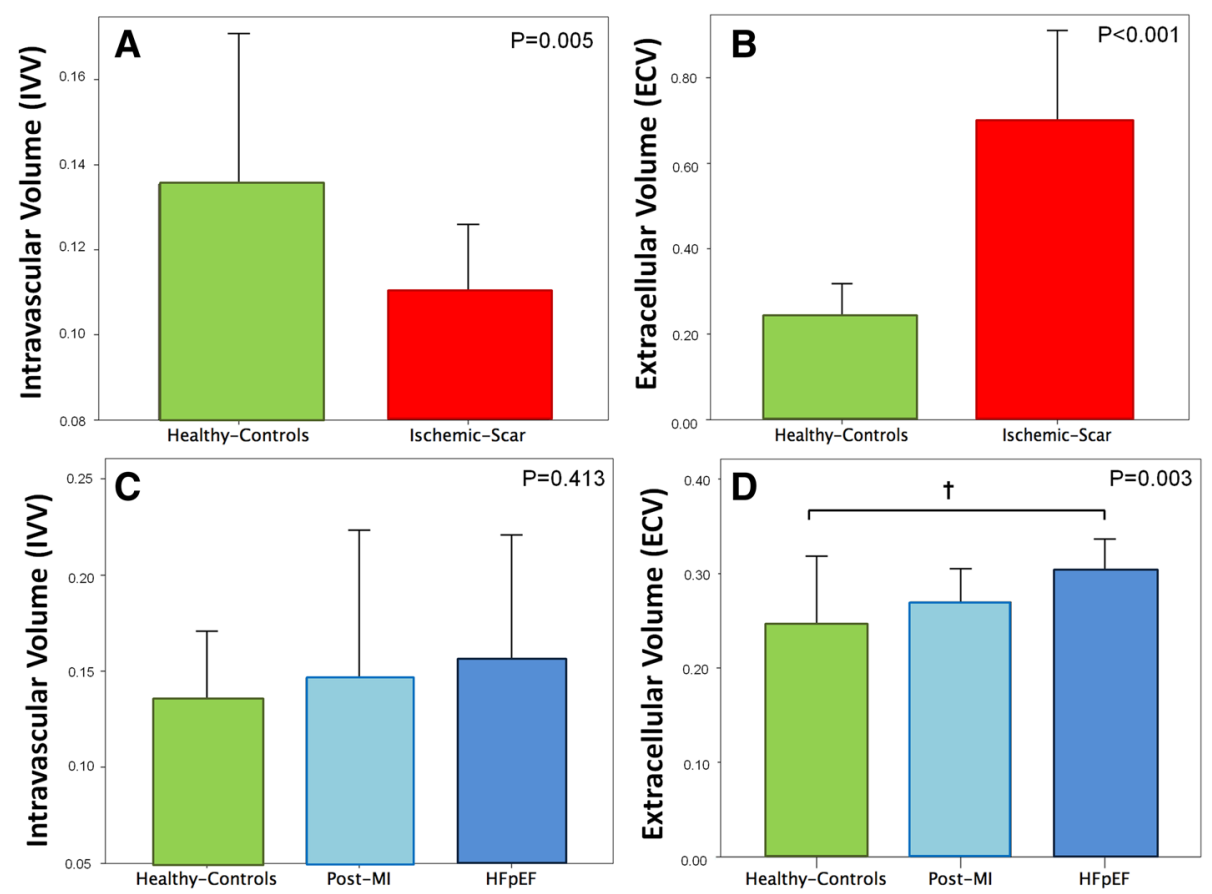

Fig. 2 Top Panel: Bars and 95\% confidence intervals of intravascular (IW; a) and extracellular (ECV; b) volumes in healthy controls (green) and ischemic scar (red). IV was reduced and ECV was strongly augmented in the ischemic scar as compared to normal myocardium. Bottom Panel: Bars and 95\% confidence intervals of IV (c) and ECV (d) in healthy (green), post-MI patients (remodeled myocardium not including the scar, light blue) and HFpEF patients (blue). Bonferroni's post-hoc analysis, $\uparrow P<0.05$

administration of an intravascular $\mathrm{CM}$, it is important to recognize that $T_{1}$-mapping measures the effect of $C M$ on proton relaxation, not reflecting directly the concentration of $\mathrm{CM}$ in the intravascular compartment. $\mathrm{Al}-$ though gadofosveset is a prevalently intravascular CM, it affects the extravascular compartment by proton (i.e. water) exchange across the vascular wall [19]. Several studies suggested a slow exchange regime for the intra extravascular water [19]. Simulations and in-vivo measurements by Donahue and co-workers also demonstrated an increasing overestimation of IVV with increasing intravascular $\mathrm{CM}$ dosages (i.e. with an increase in pre-contrast to post-contrast difference of blood relaxivity $\Delta \mathrm{R}_{1}$ ). For the slow exchange model, simulations found an overestimation of IVV by approximately $10-30 \%$ at a $\Delta R_{1}$ of 9 , which is matching the $\Delta R_{1}$ found in our study (at $3 \mathrm{~min}$ after gadofosveset injection) [19]. After IV injection, 70\% of circulating gadofosveset binds reversibly to albumin resulting in an increase in relaxivity by 5 - to 10 -fold $\left(\mathrm{r}_{1}=25 \mathrm{mmol}^{-1} \mathrm{~L}^{-1} \mathrm{~s}^{-1}\right)$ as compared with the free fraction $\left(\mathrm{r}_{1}=5 \mathrm{mmol}^{-} \mathrm{L}^{-1} \mathrm{~s}^{-1}\right)$ [20]. The unbound fraction of gadofosveset behaves as an extra-vascular extra-cellular CM, thereby increasing tissue relaxivity in the extravascular space and thus, overestimating IVV. Although we acknowledge that our protocol may lead to an overestimation of IVV by means of either mechanisms mentioned above, it should be kept in mind that overestimation is expected to occur systematically across the diverse study groups and should therefore not affect the trend of the observed pathophysiological abnormalities (e.g., increase or decrease of IVV). Taking into account that the unbound fraction of gadofosveset behaves as an extracellular extravascular CM, the overestimation of IVV is expected to be larger in those pathological conditions with an expanded extracellular compartment (i.e. larger ECV). Nevertheless, our approach showed an important decrease of IVV in the ischemic scar for which we measured a substantial expansion of the extracellular compartment in the order of $70 \%$ using an extracellular CM (ECV of $0.698 \pm 0.106$ ). Our results are in line with a study by Prech and co-workers [11], who quantified capillary density in autopsy hearts. In that study, in post-MI patients, capillary density was reduced in post-ischemic non-reperfused scar tissue by approximately $40 \%$ versus normal. In infarcted myocardium, we found a reduction of IVV of about $25 \%$ versus normal, i.e. less than that reported by Prech et al. [11]. This difference matches the expected slight overestimation of $10-30 \%$ by our method as discussed above. With this reasoning, it follows that the unbound fraction of gadofosveset is very unlikely to contribute significantly to IVV overestimation in HFpEF patients, who had only slightly increased ECV as compared to healthy 
volunteers. In summary, we set out a non-invasive CMR approach to estimate IVV by measuring $\mathrm{T}_{1}$-maps before and at $3 \mathrm{~min}$ after intravascular CM bolus injection, which was demonstrated in ischemic scar versus normal myocardium of healthy controls. Reduced IVV of chronic ischemic scar is well established in the literature, and our approach was capable to correctly gauge this key feature of dense chronic fibrotic scar.

When applying the IVV measurement to non-infarcted myocardium, IVV was not reduced in HFpEF myocardium or in non-infarcted myocardium of post-MI patients. Hence, our results do not confirm some of the previous studies in HFpEF [6, 7]. In particular, in an autopsy cohort of 124 patients who died of HFpEF, Mohammed et al. reported lower coronary microvascular density and more severe fibrosis as compared to control subjects [6]. These authors also found an inverse relationship between microvascular density and fibrosis. However, in their study the HFpEF cohort was somewhat heterogeneous being constituted by $37 \%$ of patients with a previous history of MI, and as many as $74 \%$ of patients showed LV dilatation and hypertrophy. On the other hand, in our study none of the HFpEF patients had a history of coronary artery disease, evidence of ischemia, or ischemic scar, and none was in advanced stage of heart failure. Conversely, our results are in line with the study by Prech and co-workers [11], who quantified capillary density in autopsy hearts of post-MI patients. In that study, capillary density in remote non-infarcted myocardium was not different from normal myocardium of control hearts.

While slow water exchange was addressed as a potential confounder for measuring IVV in chronic fibrotic post-ischemic scar, additional confounders should be considered when interpreting our IVV results for non-infarcted remodeled myocardium. The concept of using an intravascular CM to quantify IVV is strictly based on the assumption that the intravascular $\mathrm{CM}$ does not leak into the interstitial space. In the situation of an inflammatory state of the microcirculation of remodeled myocardium as proposed in the literature [2, 21], leaking intravascular CM would cause overestimation of IVV, in particular, if one considers the higher relaxivity of the albumin-bound fraction of Gadofosveset. To minimize this effect, in the current study $\mathrm{T}_{1}$ was measured at $3 \mathrm{~min}$. Post $\mathrm{CM}$ injection, as Pedersen et al. [20] showed maximum effect of CM leakage occurring at $30 \mathrm{~min}$ post-injection in an animal model. Elevated myocardial native, i.e. pre-contrast, $\mathrm{T}_{1}$ values result in elevated IVV according the formula we used (page 5). Puntmann et al. found elevated native $T_{1}$ of viable myocardium of post-MI patients [22] and hypertrophied myocardium [23], which correlated with outcome [22]. In agreement with these studies, we also found elevated native $T_{1}$ values of non-infarcted remodeled myocardium compared to controls (see Table 2), and this elevated native $T_{1}$ may contribute to an increase in IVV. Furthermore, the type of the $\mathrm{T}_{1}$ mapping pulse sequence may also influence the calculated $\mathrm{T}_{1}$ and thus, distribution volumes. With the MOLLI sequence we used, we were able to measure reduced IVV in scar tissue (i.e. even in tissue with massively expanded extracellular space) and in a recent study, this MOLLI sequence performed best in measuring ECV among a set of different pulse sequences in comparison to histology [24].

While we did not observe differences in IVV between healthy controls and HFpEF patients, ECV was expanded in the later, which is very likely related to an accumulation of extracellular matrix in the cardiac interstitium, since myocardial edema was excluded by normal myocardial $\mathrm{T}_{2}$ values. Accordingly, our data on ECV, i.e. on interstitial fibrosis, are in line with other studies, which reported an expanded ECV compared to healthy controls and which correlated well with markers of HFpEF severity [15]. Thus, this study is in line with others in quantifying ECV as a measure of collagen accumulation, while the current non-invasive approach to measure IVV could not detect a rarefaction of the microvasculature of hypertrophied/remodeled myocardium.

\section{Limitations}

The small number of patients included represents an important limitation. This was due to the fact that on August 2016, the production of gadofosveset was halted due to commercial reasons not permitting the recruitment of further patients. Both, ECV and IVV were not validated against endomyocardial biopsy. Rarefaction of the microvasculature in scar tissue is well established in the literature [11]. Therefore, it was deemed adequate to use scar tissue as a reference to demonstrate the feasibility of the proposed method to detect reduced IVV in myocardium. While these preliminary data indicate a potential use to probe IVV and microvascular rarefication, the introduction of new intravascular CM must be awaited to be able to further test this approach and to finally assess its potential clinical usefulness.

\section{Conclusions}

The proposed technique exploiting $\mathrm{T}_{1}$-mapping in combination with the administration of an intravascular $\mathrm{CM}$ is able to detect a reduction of the intravascular compartment in post-infarction scar tissue. However, our study findings do not support the hypothesis of microcirculatory rarefication as a patho-mechanism in the myocardium of HFpEF patients.

\footnotetext{
Abbreviations

CM: Contrast medium; CMR: Cardiovascular magnetic resonance;

ECV: Extracellular volume; HFpEF: Hear Failure with preserved ejection fraction; IW: Intravascular volume; LGE: Late gadolinium enhancement; LV: Left ventricular
} 


\section{Acknowledgements}

Not applicable.

\section{Ethics approvals and consent to participate}

The study complied with the Declaration of Helsinki and all examinations were conducted at the Lausanne University Hospital after obtaining the approval from local ethics committee. Protocole 335/14: Résonance magnétique cardiovasculaire à haute résolution avec des produits de contraste intravasculaires et extravasculaires (PB_2016-01935). Approval date: 16.10.2014.All study participants provided written informed consent. All patients and healthy-controls provided written consent for publications of their clinical data and images.

\section{Funding}

None.

\section{Availability of data and materials}

The datasets used and/or analyzed during the current study are available from the corresponding author on reasonable request.

\section{Authors' contributions}

PGM analyzed and interpreted the patients' data, was a major contributor in writing and revising the manuscript. AGP analyzed and interpreted the patients' data. GB analyzed and interpreted the patients' data. JS analyzed and interpreted the patients' data, was a major contributor in writing and revising the manuscript. All authors read and approved the final manuscript.

\section{Competing interest}

Juerg Schwitter received research support from Bayer Healthcare, Germany. Pier Giorgio Masci is supported by a grant from the Swiss National Science Foundation (32003B_159727). Anna Giulia Pavon and Gregoire Berchier declare that they do not have any competing interest.

\section{Publisher's Note}

Springer Nature remains neutral with regard to jurisdictional claims in published maps and institutional affiliations.

\section{Author details}

'Lausanne University Hospital, Cardiovascular Department, and University of Lausanne, Lausanne, Switzerland. Institute of Cardiology, S. Raffaele Hospital, Milan, Italy. ${ }^{3}$ Radiology Department, University Hospital Lausanne-CHUV, Lausanne, Switzerland.

Received: 5 February 2018 Accepted: 20 December 2018 Published online: 05 January 2019

\section{References}

1. Owan TE, Hodge DO, Herges RM, Jacobsen SJ, Roger VL, Redfield MM Trends in prevalence and outcome of heart failure with preserved ejection fraction. N Engl J Med. 2006;355(3):251-9.

2. Paulus WJ, Tschope C. A novel paradigm for heart failure with preserved ejection fraction: comorbidities drive myocardial dysfunction and remodeling through coronary microvascular endothelial inflammation. J Am Coll Cardiol. 2013;62(4):263-71.

3. Zile MR, Baicu CF, Gaasch WH. Diastolic heart failure--abnormalities in active relaxation and passive stiffness of the left ventricle. N Engl J Med. 2004; 350(19):1953-9.

4. Borbely A, van der Velden J, Papp Z, Bronzwaer JG, Edes I, Stienen GJ, Paulus WJ. Cardiomyocyte stiffness in diastolic heart failure. Circulation. 2005;111(6):774-81.

5. Borbely A, Falcao-Pires I, van Heerebeek L, Hamdani N, Edes I, Gavina C, LeiteMoreira AF, Bronzwaer JG, Papp Z, van der Velden J, Stienen GJ, Paulus WJ. Hypophosphorylation of the stiff N2B titin isoform raises cardiomyocyte resting tension in failing human myocardium. Circ Res. 2009;104(6):780-6.

6. Mohammed SF, Hussain S, Mirzoyev SA, Edwards WD, Maleszewski JJ, Redfield MM. Coronary microvascular rarefaction and myocardial fibrosis in heart failure with preserved ejection fraction. Circulation. 2015;131(6):550-9.

7. Franssen C, Chen S, Unger A, Korkmaz HI, De Keulenaer GW, Tschope C, Leite-Moreira AF, Musters R, Niessen HW, Linke WA, Paulus WJ, Hamdani N. Myocardial microvascular inflammatory endothelial activation in heart failure with preserved ejection fraction. JACC Heart Fail. 2016;4(4):312-24.
8. Wong TC, Piehler KM, Kang IA, Kadakkal A, Kellman P, Schwartzman DS, Mulukutla SR, Simon MA, Shroff SG, Kuller LH, Schelbert EB. Myocardial extracellular volume fraction quantified by cardiovascular magnetic resonance is increased in diabetes and associated with mortality and incident heart failure admission. Eur Heart J. 2014; 35(10):657-64.

9. Flett AS, Hayward MP, Ashworth MT, Hansen MS, Taylor AM, Elliott PM, McGregor C, Moon JC. Equilibrium contrast cardiovascular magnetic resonance for the measurement of diffuse myocardial fibrosis: preliminary validation in humans. Circulation. 2010;122(2):138-44.

10. Ganame J, Messalli G, Masci PG, Dymarkowski S, Abbasi K, Van de Werf F, Janssens S, Bogaert J. Time course of infarct healing and left ventricular remodelling in patients with reperfused ST segment elevation myocardial infarction using comprehensive magnetic resonance imaging. Eur Radiol. 2011;21(4):693-701.

11. Prech M, Grajek S, Marszalek A, Lesiak M, Jemielity M, Araszkiewicz A, Mularek-Kubzdela T, Cieslinski A. Chronic infarct-related artery occlusion is associated with a reduction in capillary density. Effects on infarct healing. Eur J Heart Fail. 2006:8(4):373-80.

12. Kellman $\mathrm{P}$, Wilson JR, Xue H, Ugander M, Arai AE. Extracellular volume fraction mapping in the myocardium, part 1: evaluation of an automated method. J Cardiovasc Magn Reson. 2012;14:63.

13. Kellman P, Wilson JR, Xue H, Bandettini WP, Shanbhag SM, Druey KM, Ugander M, Arai AE. Extracellular volume fraction mapping in the myocardium, part 2: initial clinical experience. J Cardiovasc Magn Reson. $2012 \cdot 14 \cdot 64$

14. Dabir D, Child N, Kalra A, Rogers T, Gebker R, Jabbour A, Plein S, Yu CY, Otton J, Kidambi A, McDiarmid A, Broadbent D, Higgins DM, Schnackenburg B, Foote L, Cummins C, Nagel E, Puntmann VO. Reference values for healthy human myocardium using a T1 mapping methodology: results from the international T1 multicenter cardiovascular magnetic resonance study. J Cardiovasc Magn Reson. 2014;16:69.

15. Duca F, Kammerlander AA, Zotter-Tufaro C, Aschauer S, Schwaiger ML, Marzluf BA, Bonderman D, Mascherbauer J. Interstitial fibrosis, functional status, and outcomes in heart failure with preserved ejection fraction: insights from a prospective cardiac magnetic resonance imaging study. Circ Cardiovasc Imaging. 2016;9(12)

16. Bondarenko O, Beek AM, Hofman MB, Kuhl HP, Twisk JW, van Dockum WG, Visser CA, van Rossum AC. Standardizing the definition of hyperenhancement in the quantitative assessment of infarct size and myocardial viability using delayed contrast-enhanced CMR. J Cardiovasc Magn Reson. 2005;7(2):481-5.

17. Hor KN, Baumann R, Pedrizzetti G, Tonti G, Gottliebson WM, Taylor M, Benson DW, Mazur W. Magnetic resonance derived myocardial strain assessment using feature tracking. J Vis Exp. 2011:(48):2356.

18. Ponikowski P, Voors AA, Anker SD, Bueno H, Cleland JG, Coats AJ, Falk V, Gonzalez-Juanatey JR, Harjola VP, Jankowska EA, Jessup M, Linde C, Nihoyannopoulos P, Parissis JT, Pieske B, Riley JP, Rosano GM, Ruilope LM, Ruschitzka F, Rutten FH, van der Meer P, 2016 ESC guidelines for the diagnosis and treatment of acute and chronic heart failure. Eur J Heart 2016:37(27): 2129-2200.

19. Donahue KM, Weisskoff RM, Chesler DA, Kwong KK, Bogdanov AA Jr, Mandeville JB, Rosen BR. Improving MR quantification of regional blood volume with intravascular T1 contrast agents: accuracy, precision, and water exchange. Magn Reson Med. 1996;36(6):858-67.

20. Pedersen SF, Thrysoe SA, Paaske WP, Thim T, Falk E, Ringgaard S, Kim WY. CMR assessment of endothelial damage and angiogenesis in porcine coronary arteries using gadofosveset. J Cardiovasc Magn Reson. 2011;13:10.

21. Puntmann VO, Carr-White G, Jabbour A, Yu CY, Gebker R, Kelle S, Hinojar R, Doltra A, Varma N, Child N, Rogers T, Suna G, Arroyo Ucar E, Goodman B, Khan S, Dabir D, Herrmann E, Zeiher AM, Nagel E, International TMCMROS. T1-mapping and outcome in nonischemic cardiomyopathy. All-Cause Mortality and Heart Failure JACC CardiovasC Imaging. 2016;9(1):40-50.

22. Puntmann VO, Carr-White G, Jabbour A, Yu CY, Gebker R, Kelle S, Rolf A, Zitzmann S, Peker E, D'Angelo T, Pathan F, Elen VS, Hinojar R, Arendt C, Narula J, Herrmann E, Zeiher AM, Nagel E. international TMCMROS. Native T1 and ECV of noninfarcted myocardium and outcome in patients with coronary artery disease. J Am Coll Cardiol. 2018;71(7):766-78. 
23. Hinojar R, Varma N, Child N, Goodman B, Jabbour A, Yu CY, Gebker R, Doltra A, Kelle S, Khan S, Rogers T, Arroyo Ucar E, Cummins C, Carr-White G, Nagel E, Puntmann VO. T1 mapping in discrimination of hypertrophic phenotypes: hypertensive heart disease and hypertrophic cardiomyopathy: findings from the international $\mathrm{T} 1$ multicenter cardiovascular magnetic resonance study. Circ Cardiovasc Imaging. 2015:8(12).

24. Child N, Suna G, Dabir D, Yap ML, Rogers T, Kathirgamanathan M, ArroyoUcar E, Hinojar R, Mahmoud I, Young C, Wendler O, Mayr M, Sandhu B, Morton G, Muhly-Reinholz M, Dimmeler S, Nagel E, Puntmann VO. Comparison of MOLLI, shMOLLLI, and SASHA in discrimination between health and disease and relationship with histologically derived collagen volume fraction. Eur Heart J Cardiovasc Imaging. 2018;19(7):768-76.

Ready to submit your research? Choose BMC and benefit from:

- fast, convenient online submission

- thorough peer review by experienced researchers in your field

- rapid publication on acceptance

- support for research data, including large and complex data types

- gold Open Access which fosters wider collaboration and increased citations

- maximum visibility for your research: over $100 \mathrm{M}$ website views per year

At BMC, research is always in progress.

Learn more biomedcentral.com/submissions 